\title{
Analytical Modeling of Sensitivity Parameters Influenced by Practically Feasible Arrangement of Bio-Molecules in Dielectric Modulated FET Biosensor
}

\author{
Rahul Das $^{1}$ (D) Ankush Chattopadhyay ${ }^{2} \cdot$ Manash Chanda $^{3} \cdot$ Chandan K. Sarkar $^{1} \cdot$ Chayanika Bose $^{1}$
}

Received: 6 September 2021 / Accepted: 13 December 2021 / Published online: 28 January 2022

(c) The Author(s), under exclusive licence to Springer Nature B.V. 2021

\begin{abstract}
In this paper, analytical modeling of a Dielectric Modulated Double Gate Field Effect Transistor (DM-DGFET) for biosensing application is presented with extensive data analysis. Firstly, the size of the nanogaps and arrangements of biomolecules in those gaps are optimized with respect to the sensitivity of the above sensor. The optimized DM-DGFET is next analyzed on the basis of its modeling and simulation. This paper addresses novel issues arising from arrangements of biomolecules, especially from practical point of view. Effect of probe placement due to steric hindrance and random nature of biomolecules, are also considered. The capacitances associated with the nanogaps occupied by biomolecules, following various arrangements, are modeled. Expressions of the threshold voltage, drain current and its sensitivity in terms of variations are also derived using the capacitance model. A comparative study of the proposed and the existing architectures is made. The influence of process variation on the sensitivity of the sensor is also studied. The results from the proposed analytical model are validated with the simulated data obtained from TCAD device simulator. In conclusion, the proposed DM-DGFET based biosensor architecture will emerge as an optimal model, very useful for the study on this field in future.
\end{abstract}

Keywords DM DGMOSFET $\cdot$ Nano-gap $\cdot$ Bio-molecules $\cdot$ Sensitivity

\section{Introduction}

Biosensor research is one of the most developing fields in last few decades. Biosensor works on the principle of detection of biomolecules and gets significant importance for clinical diagnostic, defense, and in several environmental applications. Apart from the above mentioned fields, biosensor is also having a wide range of application areas, viz., in the field of optics [1], electrochemical sensors

\section{Rahul Das}

Chayanika Bose

chayanika.bose@jadavpuruniversity.ac.in

1 ETCE Department, Jadavpur University, 188, Raja S.C. Mallick Rd, Kolkata 700032, West Bengal, India

2 ECE Department, St. Thomas' College of Engineering and Technology, 4, Diamond Harbour Road, Kidderpore, Kolkata 700023, West Bengal, India

3 ECE Department, Meghnad Saha Institute of Technology, Nazirabad Rd, Uchhepota South 24 Parganas,

Kolkata 700150, West Bengal, India
[2], nanomechanics [3] etc. However such biosensors suffer from some serious shortcomings, like requirements of costly equipment, high manufacturing cost, and low sensitivity. Semiconductor based biosensor has evolved in order to overcome these issues. Field effect transistor (FET) based biosensor has several advantages, such as, cost effectiveness, better sensing mechanism, greater precision along with higher sensitivity. Ion Sensitive Field Effect Transistor (ISFET) was invented in around 1970s. Initially, it was widely used for the detection of $\mathrm{pH}$ level in any type of solution [6-8]. The concentration of the ions in the analytes controls the performance of the device. From the deviation of the threshold voltage the concentration of ion or the $\mathrm{pH}$ value was detected [9-11]. In spite of its good performance for the detection of ionic solution or charged molecules, the sensitivity of ISFET degrades for the neutral biomolecules [12]. Therefore, researchers felt the need to design biosensor that can be equally sensitive for charged as well as neutral biomolecules. Dielectric Modulated FET (DMFET) appears as the perfect solution for this issue. In DMFET, the change in the gate capacitance occurs due to the change in dielectric constant of the materials to be tested, and (that) results in 
(the) deviation in the threshold voltage and drain current of (from) the device w.r.t. those with no material i.e. with air filled nanogaps [12]. On the basis of such deviations, the target material can be characterized. The basic 3D structure of DMFET was proposed by Lm et al. in [12]. In this type of biosensor devices, formation of a nanogap involves a thin film deposition and subsequent wet etching processes. This reduces the complications in lithographic process associated with the planar nanogap FETs [13-17]. As sensitivity is the prime concern of biosensors, search for highly sensitive biosensor is still in progress. Various unique structures have been proposed to improve the sensitivity. Narang et al. [18] proposed a DG-MOSFET based Dielectric modulated structure for better response. As this structure comprises of two nanogaps for trapping biomolecules, its sensing range is expected to be much wider.

As far as the modeling is concerned, significant developments took place in last few years. Narang et al. presented a tunnel FET based bio-sensor considering dielectric-modulation [19]. They also reported a comparative analysis between dielectric modulated FET and TFET in the context of biosensors [20]. Choi et al. modeled the nanogap-embedded FET for bio-sensor applications [21]. Bhattacharyya et al. assessed the performance of a dual-pocket vertical heterostructure tunnel FET-based biosensor [22].

In all the above articles, dielectric modulation has been considered. But, the various possible filling patterns generated by the bio-molecules trapped in nanogap are believed to influence the sensitivity of the biosensor. Unfortunately, this problem is not yet addressed.

In this work, some practical surface profiles of organic fluids appeared in biomolecule-filled nanogaps in DG
MOSFET will be considered for analytical modeling, for the first time. The silanization process of surface functionalization will be considered in this work, in which the hybridization of bio-molecules with $\mathrm{SiO}_{2}$ takes place [22]. The structure of the nanogap and its surrounding material will be optimized in order to improve the sensitivity. A detail analytical model of surface potential, threshold voltage, drain current and sensitivity will be presented considering various surface profiles of biomolecules filling the nanogaps created in both sides of the gates. Considering such four patterns (increasing/decreasing inward pattern, concave and convex pattern), the threshold voltage and ON-current sensitivity will be determined. The deviations in threshold voltage and ON-current sensitivity will also be studied. In addition, the effects of process variations on bio-sensing parameters will be analyzed in detail. The analytical modeling of the proposed device structure will be validated with the simulated results obtained from Silvaco TCAD.

\section{Device Description}

The device structure is based on traditional n-channel DGMOSFET with a dielectric modulated nanogap embedded gate insulator as shown in Fig. 1. $\mathrm{SiO}_{2}$ layer underneath the gate has divided into hydroxyl (-OH) bond and acted as a biomolecule receptor. For a better operation and sensitivity, a thin layer of $1 \mathrm{~nm} \mathrm{SiO} 2$ on the top of the nanogap is also kept untouched during the etching process. $T_{o x}, T_{G}$ and $t_{s i}$ denote the thickness of the oxide layer, gate and Si-film respectively.
Fig. 1 Cross sectional structure of a dielectric modulated double gate MOSFET (DM DGFET) with fully filled nanogap

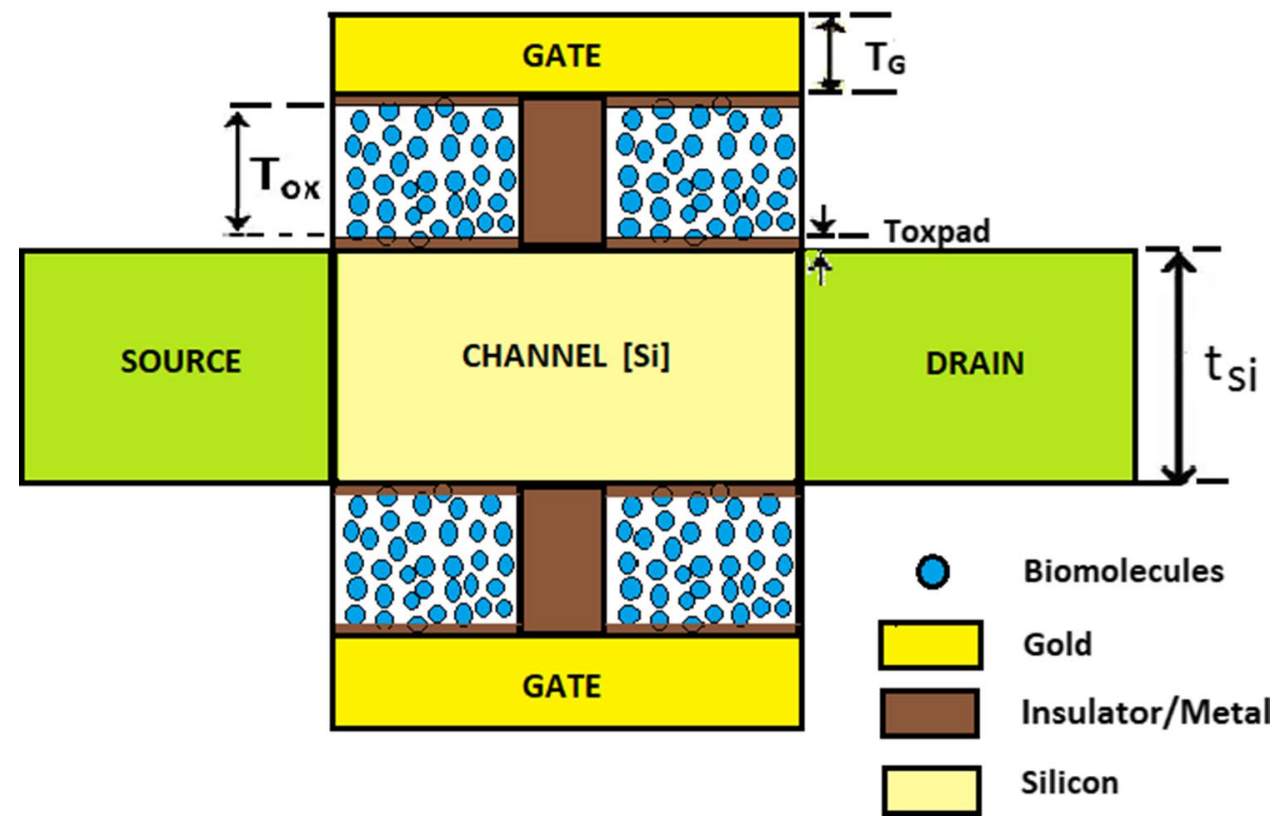




\section{Optimization}

\subsection{Material Optimization}

In past few decades, researchers realised nanogaps by partly etching out various materials used in the dielectric layer. Amongst several materials, Silicon Di-oxide $\left(\mathrm{SiO}_{2}\right)$, Hafnium Di-oxide $\left(\mathrm{HfO}_{2}\right)$, and Poly-Si are used most frequently. In this literature, a comparative analysis of these three materials, considering the biomolecules of distinct permittivities, has been performed based on the threshold voltage and current sensitivity. Most popular biomolecular materials used nowadays are APTES $(\boldsymbol{k}=\mathbf{3 . 5 7})$, biotin $(\boldsymbol{k}=\mathbf{2 . 6 3})$, Keratin $(\boldsymbol{k}=\mathbf{5}-\mathbf{1 0})$, amino acids $(\boldsymbol{k}=\mathbf{1 1}-\mathbf{2 5})$, in which the permittivity $(\boldsymbol{k})$ varies in the range of $\mathbf{2} \mathbf{- 2 5}$. Here, the materials of three different permittivity values $(\boldsymbol{k}=\mathbf{2 , 1 1 , 2 2})$ are examined in detail. Figures 2 and 3 depict that the device with $\mathrm{SiO}_{2}$ as the nanogap surrounding material yields the best performance judged on the basis of both type of sensitivities. The sensitivity increases 2 to 4 times with $\boldsymbol{k}=\mathbf{1 1 , 2 2}$ respectively for the change in surrounding materials of nanogap, i.e., $\mathrm{SiO}_{2}$ as a replacement of $\boldsymbol{P o l y}-\boldsymbol{S i}$. The value of $\Delta \boldsymbol{V}_{\boldsymbol{t h}}$ in case of $\mathrm{SiO}_{2}$ increases with the increment of permittivity of the biomolecules due to the increase in associated field lines. On the other hand, Poly-Si being a good conductor, does not cause any significant change in $V_{t h}$ due to the change of biomolecules; it has thus similar $\Delta V_{t h}$ for all $\boldsymbol{k}(=\mathbf{2 , 1 1 , 2 2})$. Therefore, the simulation results firmly establish the fact that the device with $\mathrm{SiO}_{2}$ as nanogap lining material is the best performer among the three materials for bio sensing applications. Therefore, the device with $\mathrm{SiO}_{2}$ is chosen for further optimization.

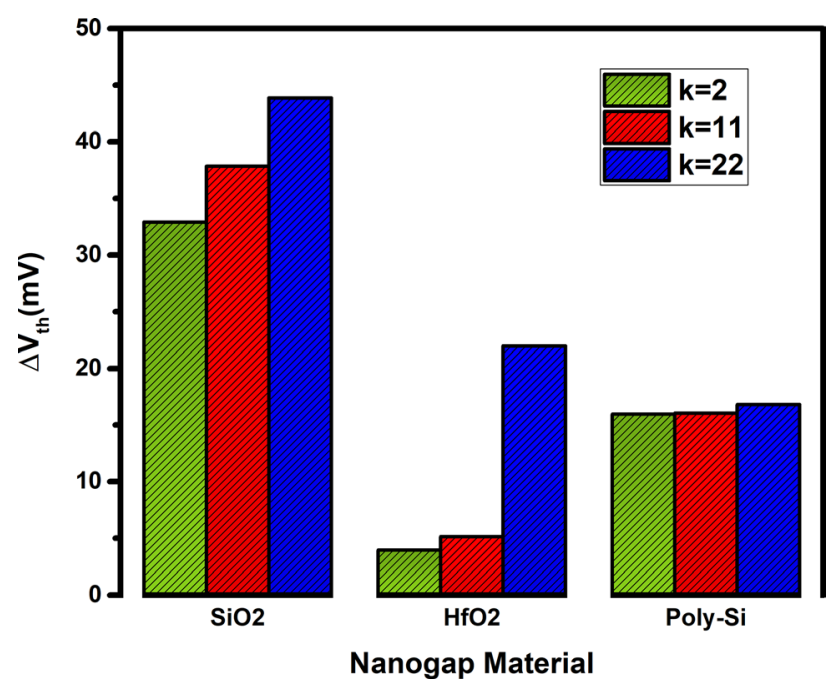

Fig. 2 Threshold voltage sensitivity analysis for different nanogap surrounding materials

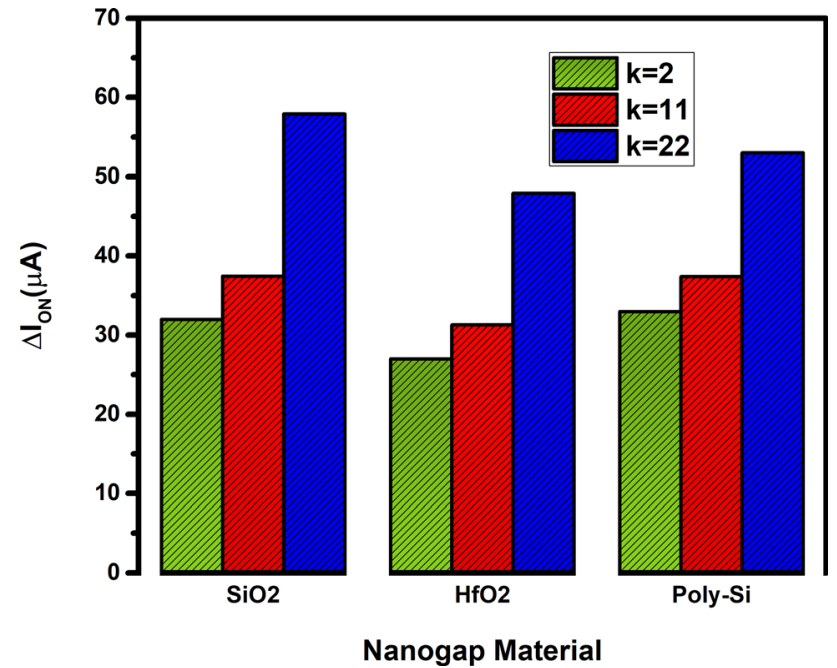

Fig. 3 ON current sensitivity analysis for different nanogap surrounding materials

\subsection{Structural Optimization}

Optimization of nanogap structure, based on its height and width, is performed in this literature for the first time. The maximum length of the nanogap in each side is chosen as one third of the gate length $(\boldsymbol{L}=\mathbf{7 5} \mathbf{n m})$. in order to maintain proper functionality. Thus a minimum of $25 \mathrm{~nm}$ I-shaped layer is maintained. The width of the nanogap in each side is considered as $25 \mathrm{~nm}, 22.5 \mathrm{~nm}$ and 20 $\mathrm{nm}$. Along with that, the height of the nanogap is also optimized with four trial values $-25 \mathrm{~nm}, 30 \mathrm{~nm}, 35$ $\mathrm{nm}$ and $40 \mathrm{~nm}$. Larger the nanogap height, greater the gate-metal separation from the channel yields poorer gate control. The ON-current and the ON-current sensitivity $\left(\Delta I_{O N}\right)$ are found to increase with the increase in permittivity of biomolecules.

The present study particularly aimed at the detection of DNA structure $(\boldsymbol{k} \sim \mathbf{1 1})$ [23]. Table 1 and Fig. 4 present the current and threshold voltage sensitivity of the biosensor with different combinations of width and height of nanogap structure fully occupied by the biomolecules. It is observed that with the increase in nanogap height, higher voltage is needed to switch ON the device and the threshold voltage sensitivity also increases $\left(\Delta \boldsymbol{V}_{t h}\right)$. Around $15 \%$ decrement in $\Delta \boldsymbol{I}_{\boldsymbol{O}}$ is observed for the increase in the height of the nanogap from $25 \mathrm{~nm}$ to $40 \mathrm{~nm}$. It is due to the narrowness of the gate dielectric and increment of the distance between channels and gate-metal, which in turn reduces the gate control over the channel. For the similar change in nanogap height, $\Delta \boldsymbol{V}_{t h}$ increases upto 2 times (width $=\mathbf{2 0} \mathbf{n m}$ ) and 2.5 times (width $=\mathbf{2 5} \mathbf{n m}$ ). Hence, it will be a healthy tradeoff, in which the device with 3 times higher $\Delta \boldsymbol{V}_{t h}$ is chosen, ignoring the negligible reduction in $\Delta \boldsymbol{I}_{\boldsymbol{O}}$. Thus, nanogap 


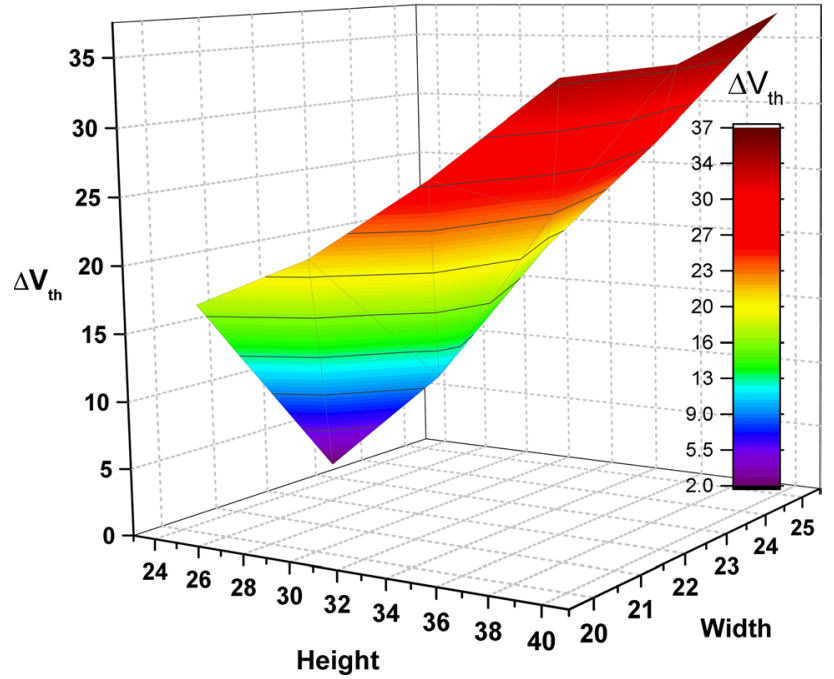

Fig. 4 3D projection of $\Delta \boldsymbol{V}_{\boldsymbol{t} \boldsymbol{h}}$ with nanogap width and height

of $25 \mathrm{~nm}$ width in both sides and $40 \mathrm{~nm}$ height has been considered for further analysis.

\subsection{Modeling of Surface Potential}

The 2D potential profile $(\psi(x, y))$ of the channel underneath the gate is determined from the Poisson's Equation, given by,

$\frac{\partial^{2} \psi(x, y)}{\partial x^{2}}+\frac{\partial^{2} \psi(x, y)}{\partial y^{2}}=\frac{q N_{a}}{\epsilon_{s i}}$

where, $q, N_{a}$ and $\epsilon_{s i}$ represent charge of an electron, doping concentration of the channel and permittivity of Si-film respectively. The solution of Eq. 1 is a parabolic function of $y,[24]$ and is expressed as follows,

$\psi(x, y)=P_{1}(x)+P_{2}(x) y+P_{3}(x) y^{2}$

The expressions of $P_{1}(x), P_{2}(x)$ and $P_{3}(x)$ are derived using the boundary conditions, as follows, i) Putting $y=0$ in Eq. 2 yields surface potential of the top surface i.e.,

$\psi(x, 0)=P_{1}(x)=\psi_{1}(x)$

ii) Electric field at the top channel is expressed as, $\left.\frac{\partial \psi(x, y)}{\partial y}\right|_{y=0}=\frac{C_{o x}\left(\psi_{1}(x)-\left(V_{G S}-\phi_{f b}\right)\right)}{\epsilon_{s i}}=P_{2}(x)$

iii) In the bottom channel, the electric field is calculated as,

$$
\begin{aligned}
\left.\frac{\partial \psi(x, y)}{\partial y}\right|_{y=t_{s i}} & =\frac{C_{o x}\left(\left(V_{G S}-\phi_{f b}\right)-\psi_{1}(x)\right)}{\epsilon_{s i}} \\
& =P_{2}(x)+2 t_{s i} P_{3}(x)
\end{aligned}
$$

Due to the symmetrical nature of the device with respect to both the gates, top and bottom channels are having same potential while operating in shorted gate mode, i.e.,

$\psi(x, 0)=\psi\left(x, t_{s i}\right)=\psi_{1}(x)$

Hence, from Eqs. 4, 5 and 6, $P_{3}(x)$ can be expressed as,

$P_{3}(x)=-\frac{P_{2}(x)}{t_{s i}}$

Therefore, the generalized expression of potential function becomes,

$$
\begin{aligned}
\psi(x, y)= & \psi_{1}(x)+\frac{C_{o x}}{\epsilon_{s i}}\left\{\psi_{1}(x)-\left(V_{G S}-\phi_{f b}\right)\right\} y \\
& -\frac{C_{o x}}{\epsilon_{s i} t_{s i}}\left\{\psi_{1}(x)-\left(V_{G S}-\phi_{f b}\right)\right\} y^{2}
\end{aligned}
$$

Putting $y=0$ in Eq. 8 yields,

$\left.\frac{\partial^{2} \psi(x, y)}{\partial x^{2}}\right|_{y=0}=\frac{d^{2} \psi_{1}(x)}{d x^{2}}$

$\left.\frac{\partial^{2} \psi(x, y)}{\partial y^{2}}\right|_{y=0}=-\frac{2 C_{o x}}{\epsilon_{s i} t_{s i}}\left\{\psi_{1}(x)-\left(V_{G S}-\phi_{f b}\right)\right\}$

Replacing the expressions of second order partial fractions from Eqs. 9a, 9b in Eq. 1 yields,

$\frac{d^{2} \psi_{1}(x)}{d x^{2}}-\frac{\psi_{1}(x)}{\alpha^{2}}=\frac{q N a}{\epsilon_{s i}}-\frac{\left(V_{G S}-\phi_{f b}\right)}{\alpha^{2}}$

where, $\alpha=\sqrt{\frac{\epsilon_{s i} t_{s i}}{C_{o x}}}$. The solution of Eq. 10 is,

\begin{tabular}{|c|c|c|c|c|c|c|c|c|}
\hline \multirow[t]{3}{*}{ Width (nm) } & \multicolumn{8}{|c|}{ Height (nm) } \\
\hline & \multicolumn{2}{|l|}{25} & \multicolumn{2}{|l|}{30} & \multicolumn{2}{|l|}{35} & \multicolumn{2}{|l|}{40} \\
\hline & $\Delta V_{t h}$ & $\Delta I_{O N}$ & $\Delta V_{t h}$ & $\Delta I_{O N}$ & $\Delta V_{t h}$ & $\Delta I_{O N}$ & $\Delta V_{t h}$ & $\Delta I_{O N}$ \\
\hline 20 & 17 & 42.7 & 21 & 40.1 & 27 & 37.7 & 34 & 35.6 \\
\hline 22.5 & 2 & 45 & 10 & 42.2 & 23 & 38.5 & 34 & 38 \\
\hline 25 & 11 & 51.2 & 18 & 48.5 & 27 & 46.1 & 37 & 43.7 \\
\hline
\end{tabular}

Table 1 Variation of $\Delta V_{t h}$ and $\Delta I_{O N}$ with nanogap width and height 
$\psi_{1}(x)=A_{1} \exp \left(\frac{x}{\alpha}\right)+A_{2} \exp \left(-\frac{x}{\alpha}\right)-A_{3}$

where,

$A_{3}=\frac{q \alpha^{2} N a}{\epsilon_{s i}}-\left(V_{G S}-\phi_{f b}\right) ; \quad$ for $0 \leq x \leq L$

\subsection{Capacitance Associated with Various Filling Patterns in Nano-Gaps}

In DM-DGFET, various bio-fluids are characterized through variations in capacitance associated with the nano-gaps filled with those fluids. In real situation, nano-gaps are hardly completely filled rather bio-fluids containing long biomolecules may follow different surface profiles within the nanogaps, and therefore offer different capacitances. Here, we will consider four basic filling patterns that ma be generated by the fluids confined in nanogaps. These are schematically presented in Fig. 5.

In order to estimate the capacitance associated with the nanogaps, we first concentrate on the smallest column containing minimum numbers of bio-molecules, as depicted in Fig. 6(a). The part of the bio-molecules occupies nanogap contribute capacitance $C_{B M}$, whereas the rest of the nanogaps contributes capacitance $C_{V A}$. Thus, the equivalent capacitance of the smallest column $\left(C_{1}=C_{V A} \| C_{B M}\right)$ can be expressed as,

$$
\begin{aligned}
C_{B M} & =\frac{5 k \epsilon_{0} \alpha_{1}}{T_{o x}} ; \quad C_{V A}=\frac{5 k_{v} \epsilon_{0} \alpha_{1}}{4 T_{o x}} \\
C_{1} & =\frac{C_{B M} C_{V A}}{C_{B M}+C_{V A}}=\frac{5 k k_{v} \epsilon_{0} \alpha_{1}}{T_{o x}\left(4 k+k_{v}\right)}
\end{aligned}
$$

Similarly, capacitance associated with other columns are calculated for various patterns of bio-molecules in a

\section{Relative permittivity of the vacant portion $=\mathrm{k}_{\mathrm{V}}$}
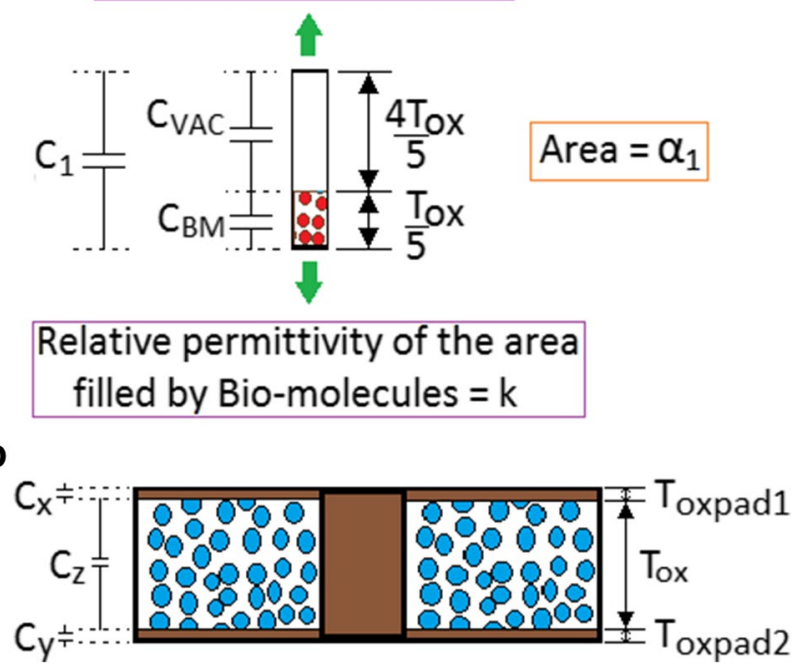

Fig. 6 a Smallest single column containing bio-molecule b General structure of nano-gap

the nano-gap. Few of them are analyzed in the following sub-sections.

\subsubsection{Patterns Increasing and Decreasing Inward Patterns}

Increasing and decreasing inward patterns are shown in Fig. 5(a). These patterns comprise of two symmetrical sections of dielectric modulated nano-gaps. Each section consists of five columns in which the height of the bio-molecule filled portion of the individual column increases with a step size of $T_{o x} / 5 . C_{i}, \alpha_{i}(i=1$ to 5$)$ denote the capacitance and area associated with the $i^{\text {th }}$ column respectively. The smallest
Fig. 5 Patterns of bio-molecule filling the nano-gaps - (a) Increasing and decreasing inward (b) Concave and convex

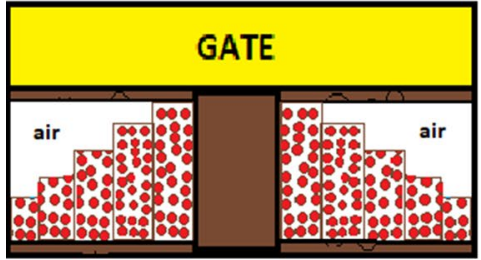

Increasing inward

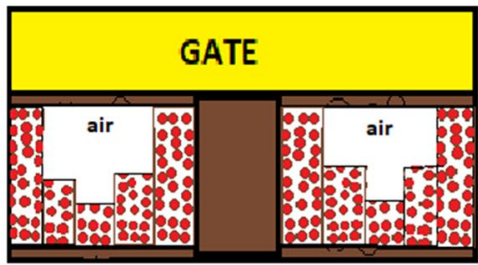

Concave

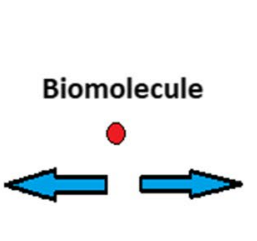

a

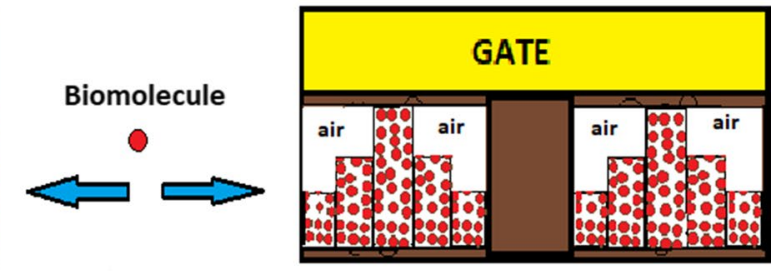

b

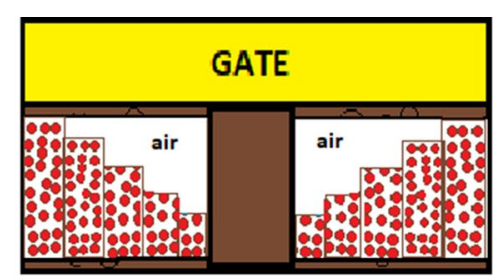

Decreasing inward

Convex 
value of $i$ corresponds to the column that consists of minimum number of bio-molecules. Therefore, the capacitances are calculated as follows,

$C_{2}=\frac{5 k k_{v} \epsilon_{0} \alpha_{2}}{T_{o x}\left(3 k+2 k_{v}\right)}$

$C_{3}=\frac{5 k k_{v} \epsilon_{0} \alpha_{3}}{T_{o x}\left(2 k+3 k_{v}\right)}$

$C_{4}=\frac{5 k k_{v} \epsilon_{0} \alpha_{4}}{T_{o x}\left(k+4 k_{v}\right)}$

$C_{5}=\frac{k \epsilon_{0} \alpha_{5}}{T_{o x}}$

Two sections of dielectric modulated nano-gaps are separated by a column and enclosed by two thin layers (top and bottom) of dielectric. The capacitance associated with the column is mathematically expressed as, $C_{c o l}=\epsilon_{o x 1} \alpha_{c o l} / T_{o x}$; where, $\epsilon_{o x 1}, \alpha_{c o l}$ are the permittivity of the dielectric and area of the column respectively. Therefore, the total capacitance $\left(C_{z}\right)$ per unit area associated with two nano-gaps and column is expressed as,

$C_{z}=\frac{2\left(\sum_{i=1}^{5} C_{i}\right)+C_{c o l}}{\alpha_{t}}$
$\alpha_{t}=2\left(\sum_{i=1}^{5} \alpha_{i}\right)+\alpha_{c o l}$

$\alpha_{t}$ is the total area associated with the nano-gaps and column. Capacitances per unit area associated with the top and bottom layers that enclose the nao-gaps, are denoted by $C_{x}$ and $C_{y}$, respectively (as shown in Fig. 6); and expressed mathematically as, $C_{x}=\epsilon_{\text {ox } 1} / T_{\text {oxpad } 1}, C_{y}=\epsilon_{\text {ox } 1} / T_{\text {oxpad } 2}$; where, $T_{\text {oxpad } 1}$ and $T_{\text {oxpad } 2}$ are the thicknesses of the two layers. Therefore, the total oxide capacitance per unit area $\left(C_{o x}\right)$ is expressed as,

$C_{o x}=\left(C_{x}\left\|C_{y}\right\| C_{z}\right)=\frac{C_{x} C_{y}+C_{y} C_{z}+C_{z} C_{x}}{C_{x} C_{y} C_{z}}$

\subsubsection{Concave and Convex Patterns}

In concave and convex pattern of dielectric modulated nanogap (as shown in Fig. 5(b)), each side consists of five columns assumed to be partly or fully filled with bio-molecules. The smallest height of bio-molecules filled column is assumed to be $T_{o x} / 3$ and the rest portion of height $2 T_{o x} / 3$ is vacant (filled by air). The height of bio-molecules increases/decreases by a step height of $T_{o x} / 3$. Assuming, the capacitance and area associated with the $i^{\text {th }}$ column are $C_{i}, \alpha_{i}(i=1$ to 3 ) respectively. The value of $i$ increases with the height of the trapped bio-molecules. Thus, the total capacitance per unit area associated with concave pattern is expressed as,

$C_{z}=\frac{2\left(C_{1}+2 C_{2}+2 C_{3}\right)+C_{c o l}}{\alpha_{t}}$

$\alpha_{t}=2\left(\alpha_{1}+2 \alpha_{2}+2 \alpha_{3}\right)+\alpha_{c o l}$

$C_{1}=\frac{3 k k_{v} \epsilon_{0} \alpha_{1}}{T_{o x}\left(2 k+k_{v}\right)}$

$C_{2}=\frac{3 k k_{v} \epsilon_{0} \alpha_{2}}{T_{o x}\left(k+2 k_{v}\right)}$

$C_{3}=\frac{k \epsilon_{0} \alpha_{3}}{T_{o x}}$

For convex pattern, total capacitance per unit area is calculated as,

$C_{z}=\frac{2\left(2 C_{1}+2 C_{2}+C_{3}\right)+C_{c o l}}{\alpha_{t}}$

$\alpha_{t}=2\left(2 \alpha_{1}+2 \alpha_{2}+\alpha_{3}\right)+\alpha_{c o l}$

where $C_{1}, C_{2}, C_{3}$ can be determined from Eq. 16. Putting the expressions of $C_{z}$ from Eqs. 16 and 17 in Eq. $15 C_{o x}$ for different patterns can be obtained.

\subsection{Expressions of Coefficients}

Expressions of constants such as, $A_{1}, A_{2}$ can be derived from the continuity equations and built-in potential developed at the interface of two different regions. Built-in potentials developed at the source-channel and drain-channel interfaces are denoted by $P_{4}$ and $P_{5}$ respectively. The expressions of $P_{4}$ and $P_{5}$ are given by,

$P_{4}=\frac{k T}{q} \ln \left(\frac{N a N_{s}}{n_{i}^{2}}\right)$

$P_{5}=\frac{k T}{q} \ln \left(\frac{N a N_{s}}{n_{i}^{2}}\right)+V_{D}$

The continuity equations of potential at the source/drain channel interface are expressed as,

$\left.\psi_{1}(x)\right|_{x=0}=P_{4}$

$\left.\psi_{1}(x)\right|_{x=L}=P_{5}$

From Eqs. 11, 19a and 19b, the expressions of $A_{1}$ and $A_{2}$ are derived as,

$A_{1}=P_{4}+A_{3}+A_{2}$ 
$A_{2}=\frac{\left(P_{4} \exp \left(\frac{L}{\alpha}\right)-P_{5}\right)+A_{3}\left(\exp \left(\frac{L}{\alpha}\right)-1\right)}{\exp \left(\frac{L}{\alpha}\right)-\exp \left(-\frac{L}{\alpha}\right)}$

\subsection{Threshold Voltage $\left(V_{t h}\right)$}

Threshold voltage is defined as the minimum gate-to-source voltage to make the surface potential equal to the twice of the Fermi potential $\left(\phi_{f, s i}\right)$ of the channel. It is expressed mathematically as [25],

$\left.\psi_{1}\left(x=x_{\min }\right)\right|_{V_{G S}=V_{t h}}=2 \phi_{f, s i}$

where, $x_{\min }$ is the location of the minimum surface potential from the source-channel interface [26], obtained from the following equation,

$\left.\frac{d \psi_{1}(x)}{d x}\right|_{x_{\min }}=0$

Further calculation yields,

$\exp \left(\frac{x_{\min }}{\alpha}\right)=\sqrt{\frac{A_{1}}{A_{2}}}$

From Eqs. 21 and 23 it is derived as,

$2 \phi_{f, s i}=2 \sqrt{A_{1} A_{2}}-A_{3}$

Various related co-efficients are expressed as,

$A_{2}=P_{6}-V_{G S} P_{7}$

$A_{1}=P_{8}-V_{G S} P_{9}$

$A_{3}=P_{10}-V_{G S}$

The expressions of $P_{i}$ for ( $i=6$ to 10$)$ are specified in Appendix. Putting the expressions of $A_{1}, A_{2}$ and $A_{3}$ from Eq. 25 into Eq. 24 yields a quadratic equation of $V_{G S}$ as,

$P_{11} V_{G S}^{2}+P_{12} V_{G S}+P_{13}=0$

solution of which yields the expression of threshold voltage,

$V_{G S}=\frac{-P_{12} \pm \sqrt{P_{12}^{2}-4 P_{11} P_{13}}}{2 P_{11}}$

The expressions of $P_{11}, P_{12}$ and $P_{13}$ are shown in Appendix.

\subsection{Sensitivity $\left(S_{b i o}\right)$}

Sensitivity(S) has been defined as the change in the threshold voltage with biomolecules trapped in nanogap cavities with respect to the empty cavity [18], given as,
$S_{\text {bio }}=\left(V_{T(\text { empty })}-V_{T(\text { bio })}\right) / V_{T(e m p t y)}$

It is found that the $S_{b i o}$ increases with the increase in the height of the cavity as its permit larger number of molecules to get into it. The literature [18] concludes that larger molecules offer better sensitivity. Streptavidin and other larger molecule of size around 6-7 $\mathrm{nm}$ is the best molecule to fit in this device. It is also reported that the device with completely full cavity offers better sensitivity. However, in the full cavity structure as the gate is floating, it should have fabrication issues. It requires body support during fabrication, that in turn nullify the effect of down-scaling.

\subsection{Drain Current $\left(I_{D S}\right)$}

Drain current model of the DG MOSFET based structure can be represented for its various operating regions, i.e., subthreshold, linear and saturation. Liang et al. in [27] had presented the subthreshold current model which can be used to obtain the $I_{D S, s u b}$. In linear and saturation regions of operation, the drain current model proposed in [28] is used in the present study as follows,

$$
\begin{aligned}
I_{D S, \text { sub }}= & \frac{k T W \mu_{n} n_{i}\left(1-e^{\left(-V_{D S}\right) / V_{T}}\right)}{\int_{0}^{L} \frac{d x}{\int_{0}^{I_{i}} e^{\psi_{1}(x) / V_{T}} d y}} \\
I_{D S, \text { lin }}= & {\left[\left(V_{G S}-V_{t h}\right)^{\delta} V_{D S}-\frac{1}{2} V_{D S}^{2}\right] } \\
& {\left[\frac{2 \mu C_{o x} W}{\left(L-\lambda_{c}-\frac{V_{D S}}{E_{C}}\right)}+\lambda_{a} \frac{2 C_{o x} W}{\left(L-\lambda_{c}\right)^{2}}\right] } \\
I_{D S, \text { sat }}= & {\left[\beta\left(V_{G S}-V_{t h}\right)^{\delta} V_{D s a t}-\frac{1}{2} V_{D s a t}^{2}\right] } \\
& {\left[\frac{2 \mu C_{o x} W}{\left(L-\lambda_{c}-\frac{V_{D S}}{E_{C}}\right)}+\lambda_{a} \frac{2 C_{o x} W}{\left(L-\lambda_{c}\right)^{2}}\right] }
\end{aligned}
$$

where, $\lambda_{a}$ and $\lambda_{c}$ represent the effect of velocity overshoot [29] and channel length modulation factor [30] respectively. The saturation voltage $\left(V_{D s a t}\right)$ is calculated from the formula, $V_{D s a t}=\frac{V_{G S}-V_{t h}}{1+\frac{V_{G S}-V_{t h}}{L E_{C}}}$ with $E_{C}=\frac{2 v_{s a t}}{\mu}$, where, $v_{s a t}$ denotes the saturation velocity of electron [28]. Values of $\beta, \delta$ are given in $[31,32]$.

\section{Model Validation}

In this section, the proposed model is compared with the simulated data. The transfer characteristics of cocave convex and increasing decreasing patterns considering both the model and simulated data are shown in Fig. 7. Perfect 

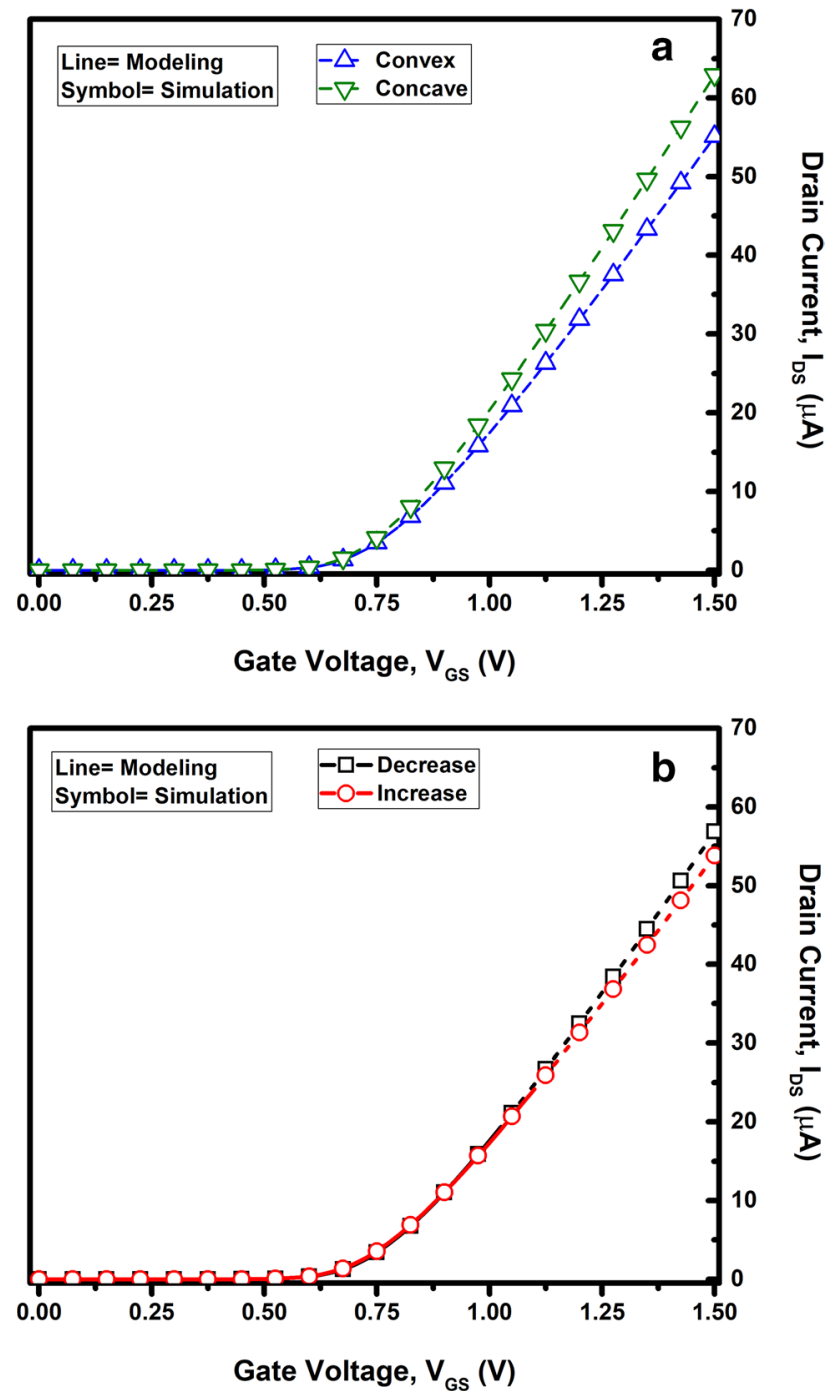

Fig. 7 Model validation for a Cocave and convex pattern, b Inward increasing and decreasing pattern, with $V_{D S}=1 \mathrm{~V}$

matching between the two is observed, which validates the proposed model.

\section{Results and Discussions}

The results obtained from the modeling and simulations (using SILVACO TCAD) of the DM DGFET with Fully Filled Nanogap considering the effects of different biomolecular patterns have been thoroughly analyzed. The threshold voltage and the ON-current sensitivity have been calculated for four different patterns of bio-molecules confined in the nano-gaps. The deviation of the above parameters from the fully filled structure has also been calculated. In the mirror image pattern, the top and bottom nanogaps are assumed to be exactly same.

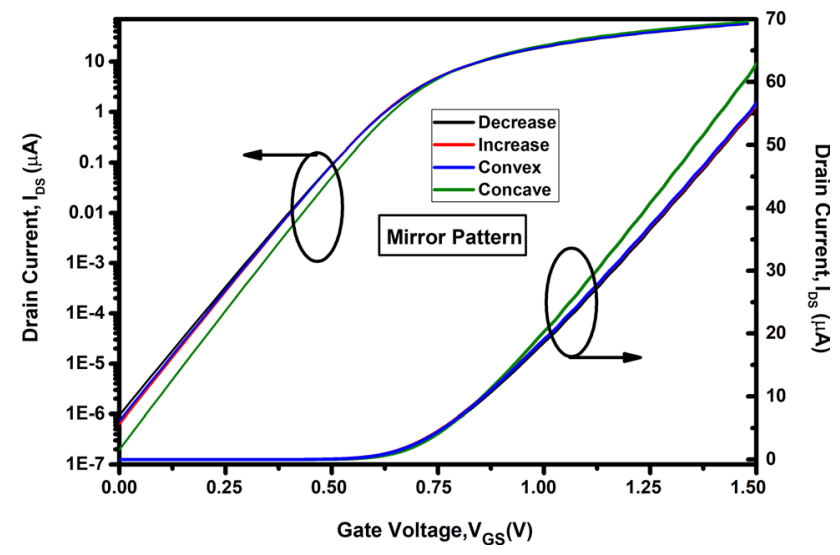

Fig. 8 Transfer characteristic study for different patterns

In Fig. 8, the transfer characteristics of four different patterns have been depicted. It is clearly observed from the figure that the concave pattern exhibits the highest $\mathrm{ON}$-current leading to a deviation margin of $13 \%$. It also produces the lowest OFF-current. The other three patterns generate 6-9 times larger OFF-current than the concave counterpart.

Although, the fully filled structure of DG-MOSFET based bio-sensor has been analyzed in various literatures, partially (half and quarterly) filled nano-gap may describe the real situation better, which needs special attention. In partially filled nano-gap structure, mirror-like pattern of top and bottom nanogaps has been studied in various reports. However, this pattern is totally dependent on the orientation of biosensor arising from its placing. It may vary depending on placement of the sensor in the bio-molecular solution. For this reason, along with conventional mirror pattern in the bottom nanogap, the same pattern both in the top and bottom nanogaps has also been tested. The mirror type pattern architecture is termed as Dielectric modulated DGMOSFET with Mirror Structure (DM DGMOSFET-MS), whereas the same type architecture is termed as Dielectric modulated DGMOSFET with Same Structure (DM DGMOSFET-SS); these are depicted in Fig. 9(a), (b), respectively. Both of these nano-gap architectures are analyzed in detail and the values of various parameters are given in Tables 2 and 3, as follows.

In Fig. 10, the transfer characteristic of the DM-DGMOSFET-SS is presented. It also exhibits a similar trend as that of the DM-DGMOSFET-MS. The concave pattern yields the lowest OFF-current and highest $\mathrm{ON}$-current in both the device architectures.

Figure 11 depicts the comparative analysis of DMDGMOSFET-MS and DM-DGMOSFET-SS in terms of $\Delta \boldsymbol{V}_{\boldsymbol{t} \boldsymbol{h}}$ for different patterns. It is quite clear from the figure that DM-DGMOSFET-SS yields far better results than the DM-DGMOSFET-MS in most of the cases. As far as 


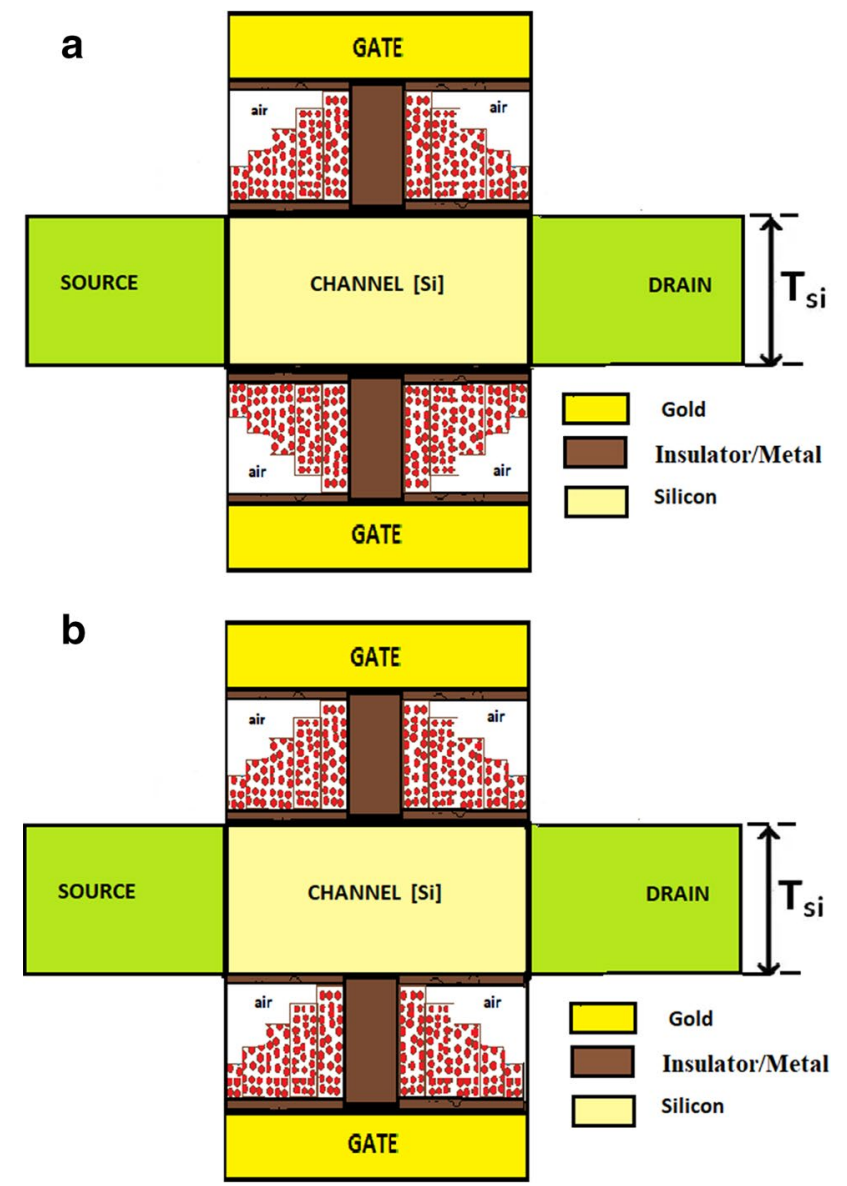

Fig. 9 Pattern depicting DM DGMOSFET a) MS b) SS structure

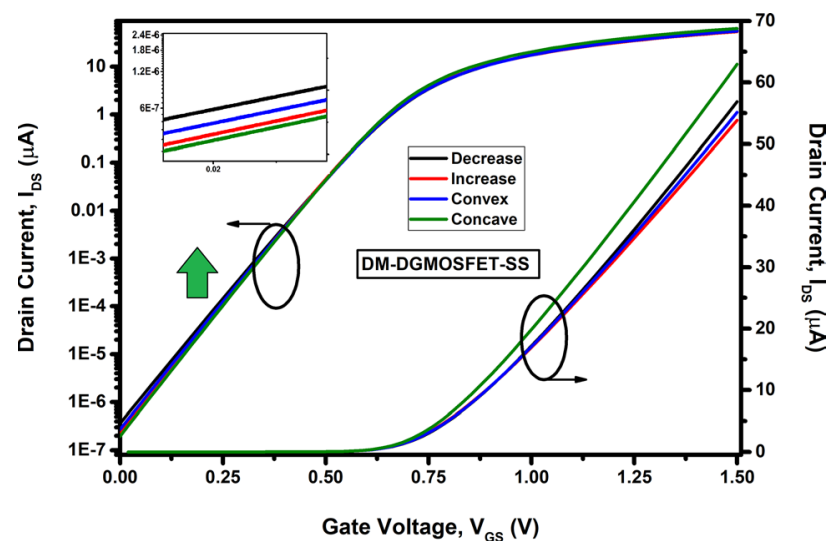

Fig. 10 Transfer characteristic of different patterns with the same type of architecture

the decreasing pattern is concerned, DM-DGMOSFET-SS outerforms yielding 30 times higher $\Delta \boldsymbol{V}_{\boldsymbol{t} \boldsymbol{h}}$. In addition, the concave and convex pattern shows $400 \%$ and $100 \%$ higher value for DM-DGMOSFET-SS, respectively.

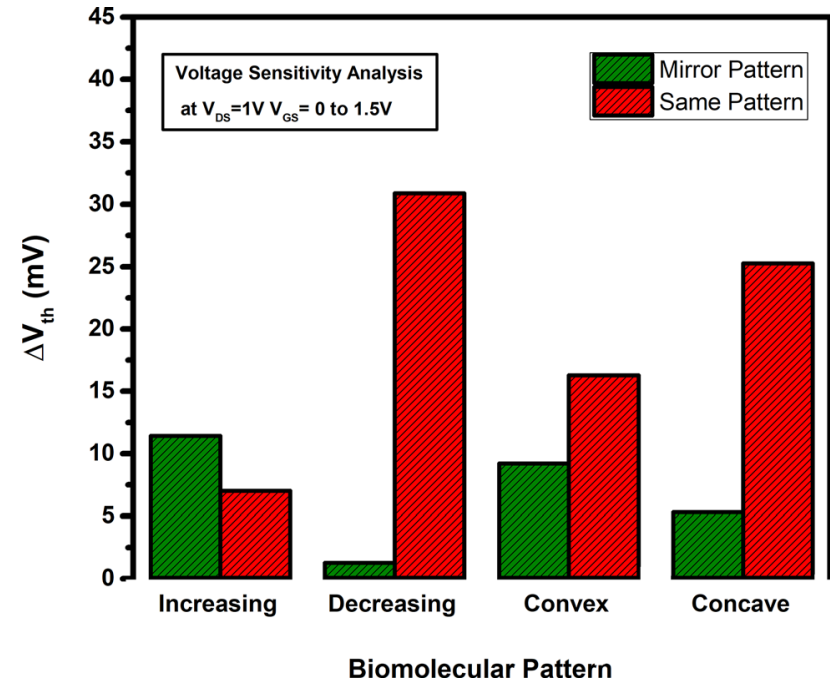

Fig. 11 Threshold voltage sensitivity $\left(\Delta V_{t h}\right)$ for different patterns

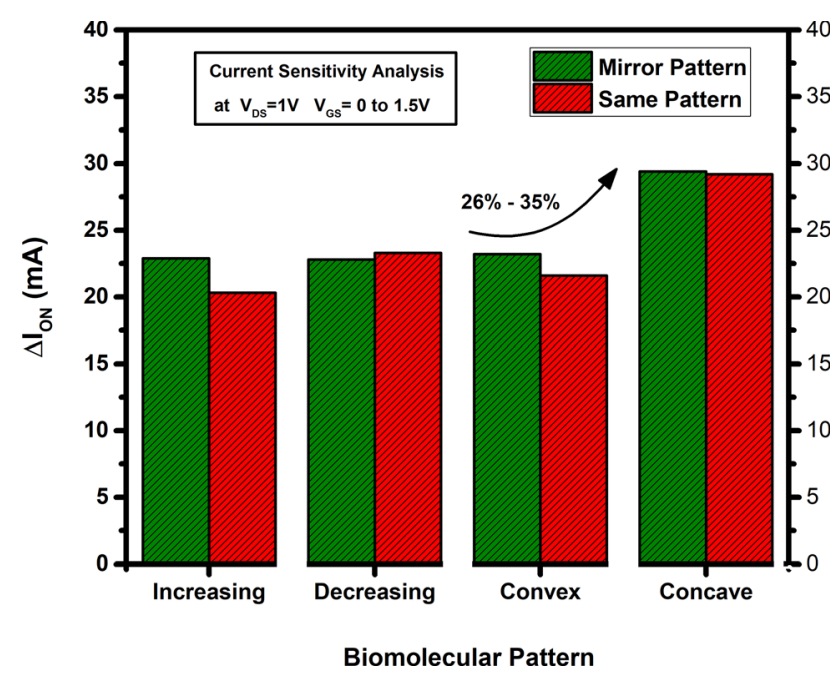

Fig. 12 ON-current sensitivity $\left(\Delta I_{O N}\right)$ comparison for different patterns

ON-Current Sensitivity $\left(\Delta \boldsymbol{I}_{\boldsymbol{O}}\right)$ for the same type of devices with similar patterns is depicted in Fig. 12. It is found that both the architecture offer almost the same sensitivity. However, in between four patterns, the concave pattern for both DM-DGMOSFET-MS and DM-DGMOSFETSS shows almost $26-35 \%$ higher sensitivity compared to other three patterns.

Researches had mostly considered the ideal situation where the nano-gap is fully filled. However, in reality due to the steric hindrance, probe placement and other fabrication disorders, the nanogap may not be completely filled. The deviation of the threshold voltage $\left(\Delta V_{t h}\right)$ in reference to the device with a full-filled cavity is termed as "error", which is 


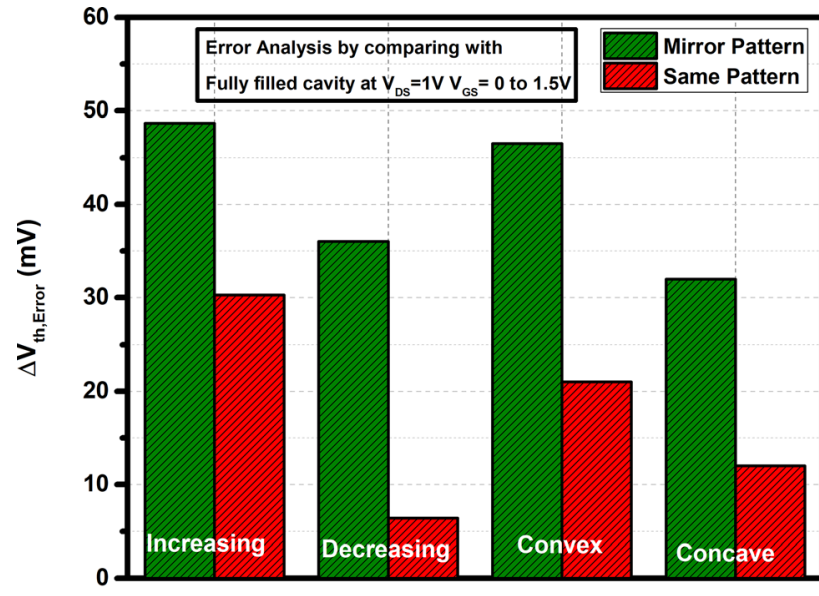

Biomolecular Pattern

Fig. 13 Comparison of error in threshold voltage sensitivity $\left(\Delta V_{t h, E r r o r}\right)$ calculated with respect to fully filled structure

essential in the domain of bio-sensor application. The biomolecular pattern having the least error is supposed to be the most perfect i.e., closest to the ideal case. Depending upon the highest sensitivity and lowest error values, a trade-off should be done, which can be used in future.

Figure 13 illustrates the $\boldsymbol{\Delta} \boldsymbol{V}_{\text {th } \text { Error }}$ of DM-DGMOSFET-MS and DM-DGMOSFET-SS devices for four different patterns. From the figure, it is quite clear that the DM-DGMOSFET-SS follows the same trend as that of the DM-DGMOSFET-MS, but the magnitude of error is almost $60-65 \%$ lower for the former device, which is highly appreciable. Among the four patterns, decreasing and concave patterns yield lower $\Delta \boldsymbol{V}_{\boldsymbol{t h}, \boldsymbol{E r r o r}}$ values in both the cases. The concave pattern showcases the minimum value for DMDGMOSFET-MS, whereas the decreasing pattern exhibits the minimum value for DM-DGMOSFET-SS. However, their difference is almost negligible. The concave pattern shows a maximum of $33 \%$ and $60 \%$ lower $\Delta V_{t h \text { Error }}$ in case of DM-DGMOSFET-MS and DM-DGMOSFET-SS, respectively. However, the decreasing pattern shows a negligible $4 \mathrm{mV}$ lower error than the concave pattern when used in DM-DGMOSFET-SS configuration.

In Fig. 14, $\Delta \boldsymbol{I}_{O N, \text { Error }}$ has been plotted for different patterns. It is evident from the figure that the concave pattern yields the lowest error value, around $30-40 \%$ lower from its other counterparts for both DM-DGMOSFET-MS and DM-DGMOSFET-SS. The two values of $\Delta \boldsymbol{I}_{\boldsymbol{O N}, \text { Error }}$ for the concave pattern are more-or-less equal. The typical values of threshold voltage, ON-current and its sensitivity for both DM-DGMOSFET-MS and DM-DGMOSFET-SS considering all four patterns are given in Tables 2 and 3 respectively.

It has been found from the study that DM-DGMOSFETSS depicts better performance than DM-DGMOSFET-MS in

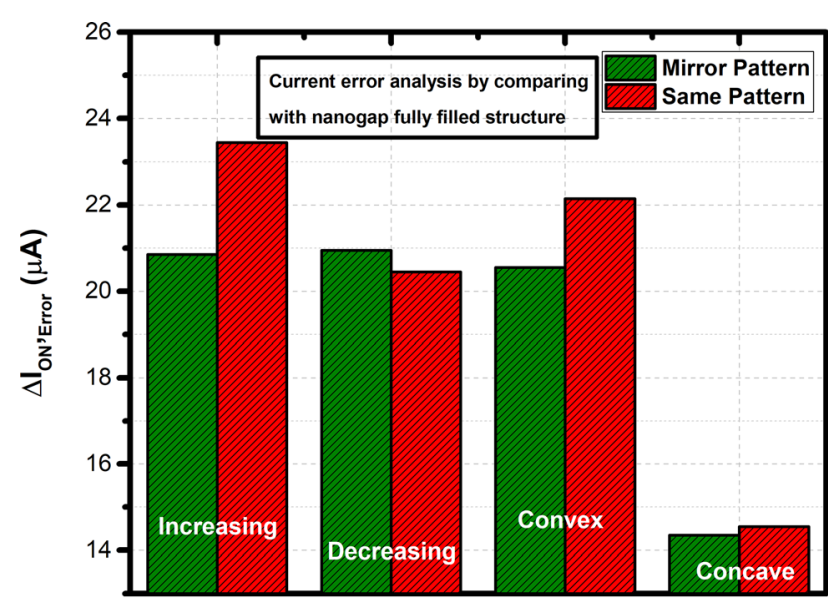

Biomolecular Pattern

Fig. 14 Comparison of error in ON current sensitivity $\left(\Delta \boldsymbol{I}_{\boldsymbol{O N}, \boldsymbol{E r r o r}}\right)$ calculated with respect to Fully filled structure

every aspect, and the concave pattern emerges as the preferable one in most of the cases. Therefore, DM-DGMOSFETSS with concave pattern has been chosen for further studies.

\subsection{Effect of Process Variation on Biosensing Parameters}

So far, the study has been carried out assuming the symmetrical device structure. It is evident that the DMDGMOSFET-SS with concave biomolecular pattern and optimised nanogap offers the best performance in terms of sensitivity and error analysis. In contemporary researches, mainly in $\mathrm{H}$. Im et al. [12], it is stated that the nanogap used in the biosensing process, is an outcome of sensible wet etching procedure. Although the fabrication process are performed with extreme precision, the influence of process variation can not be made totally absent. In this section, a structure which may appear due to the variation in the wet etching process has been studied. Four types of structural variations due to the variation in etching process are considered below.

(a) Nanogaps wider in drain side under both top and bottom gates,

(b) Nanogaps wider in source side under both top and bottom gates,

(c) Nanogaps wider in drain side under only one gate,

(d) Nanogaps wider in source side under only one gate.

The structures are schematically presented in Fig. 15. All these structures are considered to be Asymmetric as they deviate from the ideal symmetric structure. In case 
Table 2 Sensitivity and error analysis for mirror pattern

\begin{tabular}{|c|c|c|c|c|c|c|c|c|c|c|}
\hline \multirow[t]{3}{*}{ Pattern } & \multicolumn{10}{|c|}{ Mirror pattern (DGMOSFET-MS) } \\
\hline & \multicolumn{2}{|c|}{ Device of interest } & \multicolumn{2}{|c|}{ Air-filled } & \multicolumn{2}{|c|}{ Sensitivity } & \multicolumn{2}{|c|}{ Fully filled } & \multicolumn{2}{|l|}{ Error } \\
\hline & $\begin{array}{l}V_{t h} \\
(\mathrm{mV})\end{array}$ & $\begin{array}{l}I_{O N} \\
(\mu \mathrm{A})\end{array}$ & $\begin{array}{l}V_{t h} \\
(\mathrm{mV})\end{array}$ & $\begin{array}{l}I_{O N} \\
(\mu \mathrm{A})\end{array}$ & $\begin{array}{l}\Delta V_{t h} \\
(\mathrm{mV})\end{array}$ & $\begin{array}{l}\Delta I_{O N} \\
(\mu \mathrm{A})\end{array}$ & $\begin{array}{l}V_{t h} \\
(\mathrm{mV})\end{array}$ & $\begin{array}{l}I_{O N} \\
(\mu \mathrm{A})\end{array}$ & $\begin{array}{l}\Delta V_{t h} \\
(\mathrm{mV})\end{array}$ & $\begin{array}{l}\Delta I_{O N} \\
(\mu \mathrm{A})\end{array}$ \\
\hline Increasing & 277.7 & 56.4 & 289.11 & 33.5 & 11.41 & 22.9 & 326.4 & 77.25 & 48.7 & 20.85 \\
\hline Decreasing & 290.39 & 56.3 & & & 1.27 & 22.8 & & & 36.02 & 20.95 \\
\hline Convex & 279.9 & 56.7 & & & 9.21 & 23.2 & & & 46.5 & 20.55 \\
\hline Concave & 294.4 & 62.9 & & & 5.31 & 29.4 & & & 31.9 & 14.35 \\
\hline
\end{tabular}

Table 3 Sensitivity and error analysis for DGMOSFET-SS

\begin{tabular}{|c|c|c|c|c|c|c|c|c|c|c|}
\hline \multirow[t]{3}{*}{ Pattern } & \multicolumn{10}{|c|}{ Similar pattern (DGMOSFET-SS) } \\
\hline & \multicolumn{2}{|c|}{ Device of interest } & \multicolumn{2}{|c|}{ Air-filled } & \multicolumn{2}{|c|}{ Sensitivity } & \multicolumn{2}{|c|}{ Fully filled } & \multicolumn{2}{|l|}{ Error } \\
\hline & $\begin{array}{l}V_{t h} \\
(\mathrm{mV})\end{array}$ & $\begin{array}{l}I_{O N} \\
(\mu \mathrm{A})\end{array}$ & $\begin{array}{l}V_{t h} \\
(\mathrm{mV})\end{array}$ & $\begin{array}{l}I_{O N} \\
(\mu \mathrm{A})\end{array}$ & $\begin{array}{l}\Delta V_{t h} \\
(\mathrm{mV})\end{array}$ & $\begin{array}{l}\Delta I_{O N} \\
(\mu \mathrm{A})\end{array}$ & $\begin{array}{l}V_{t h} \\
(\mathrm{mV})\end{array}$ & $\begin{array}{l}I_{O N} \\
(\mu \mathrm{A})\end{array}$ & $\begin{array}{l}\Delta V_{t h} \\
(\mathrm{mV})\end{array}$ & $\begin{array}{l}\Delta I_{O N} \\
(\mu \mathrm{A})\end{array}$ \\
\hline Increasing & 296.13 & 53.8 & 289.12 & 33.5 & 7.01 & 20.3 & 326.4 & 77.25 & 30.27 & 23.45 \\
\hline Decreasing & 320 & 56.8 & & & 30.88 & 23.3 & & & 6.4 & 20.45 \\
\hline Convex & 305.4 & 55.1 & & & 16.28 & 21.6 & & & 21 & 22.15 \\
\hline Concave & 314.4 & 62.7 & & & 25.27 & 29.2 & & & 12 & 14.55 \\
\hline
\end{tabular}

of (a) and (b), asymmetries in the nanogaps is considered under identical gates. Such structures are therefore, termed here as Aligned ones. Other two cases, i.e. (c) and (d) lack such symmetry and are designated as Misaligned structures.

Firstly, the sensitivity parameters of the above structures are investigated with the optimized nanogaps, each filled with biomolecules following the concave pattern as described earlier. As both the gates are shorted, variation in gate bias will equally influence the nanogaps under top and bottom gates.

In Fig. 16, the threshold voltage sensitivity $\left(\Delta \boldsymbol{V}_{\boldsymbol{t}}\right)$ of the four devices is exhibited. The figure suggests that the device in Fig. 15(a) and (c), i.e. the devices with wider nanogap in drain side offer $\sim 100 \%$ and $\sim 40 \%$ higher $\boldsymbol{\Delta} \boldsymbol{V}_{\boldsymbol{t} \boldsymbol{h}}$ for aligned structure and misaligned structures respectively than its source side counterpart. Therefore, more the vertical $\mathrm{SiO}_{2}$ layers (shaded Blue in Fig. 15) shift towards source end, stronger gate control and in turn, a higher sensitivity for a biosensor are achieved.

Figure 17 depicts the variation in $\Delta \boldsymbol{I}_{\boldsymbol{O N}}$ for all the above structures. However, the variations in this biosensing parameter are less than $10 \%$, and thus not much significant. It may be concluded that $\Delta \boldsymbol{I}_{\boldsymbol{O}}$ is quite insensitive to process variation related to formation of the nanogap cavities. Results related to Figs. 16 and 17 are presented in Table 4.

\section{Conclusion}

In this paper, an extensive study has been carried out on Dielectric Modulated DGFET to view its suitability as a biosensing device by modifying the structure based on the orientation of the biomolecules within the nanogaps. A novel approach has been made by optimizing the device with respect to the nanogap forming material along with the height and width of it. $\mathrm{SiO}_{2}$ emerges as the best one for creating the nanogaps by obtaining 200-400\% $\Delta V_{t h}$ than its nearest contender. The largest nanogap height (40 $\mathrm{nm})$ and width $(25 \mathrm{~nm})$ taken for optimization, have been selected based on the sensitivity. The effect of different profiles arising from distribution of biomolecules in nanogap cavities has also been studied in this paper. A mirror type profile (DM-DGFET-MS) and the same type profile (DMDGFET-SS) have been studied. Results show that for both DM-DGFET-MS and DM-DGFET-SS, concave surface profile of biomolecular solution in the nanogap is having respectively $26 \%$ and $35 \%$ better sensitivity than the other cases studied here. Its preformation deviation ( $\Delta V_{t h \text { Error }}$, $\left.\Delta I_{O N, E r r o r}\right)$ from ideal sensor with fully-filled nanogaps has been also calculated and concave profile yields $33 \%$ lower error value than its nearest counterpart for both DMDGFET-MS and DM-DGFET-SS configurations. Therefore, the study suggests that this type of FET based biosensor can be a good option for the solution, which follows a concave 

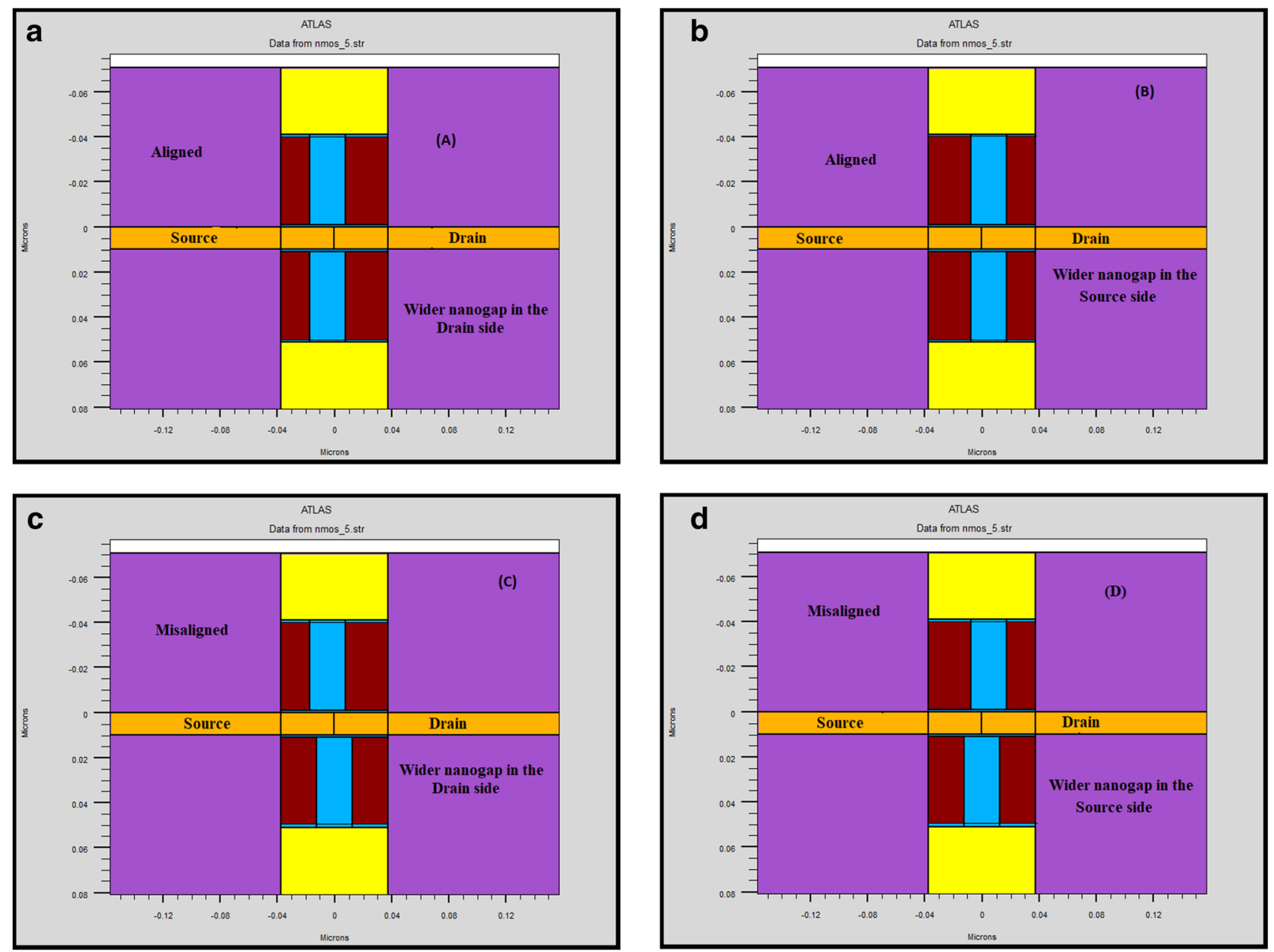

Fig. 15 Asymmetric structures: (a) Wider drain- nanogap under both top and bottom gate, (b) Wider source-nanogap under both top and bottom gate, (c) Wider drain-nanogap under only one gate, (d) Wider source- nanogap under only one gate

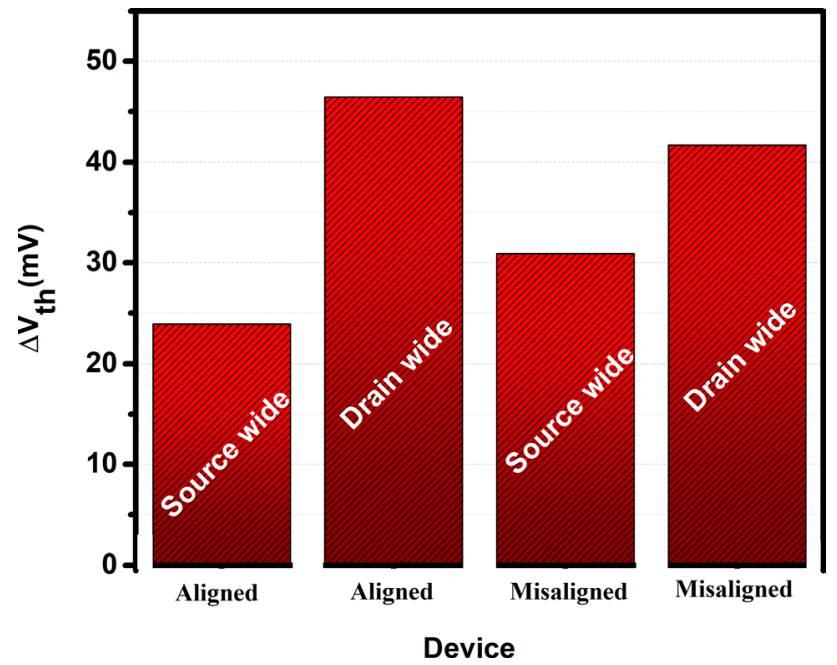

Fig. 16 Threshold voltage sensitivity $\left(\Delta V_{t h}\right)$ for four asymmetric devices

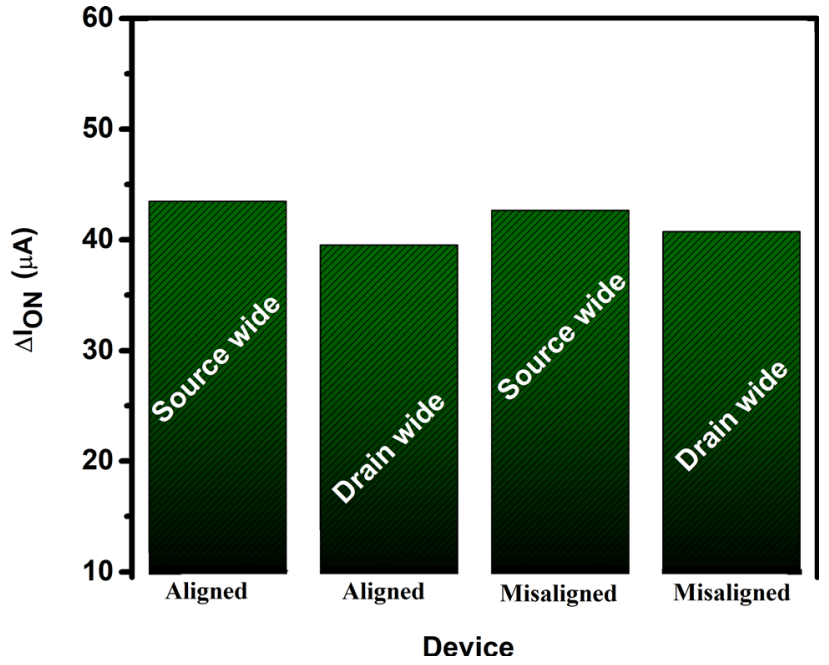

Fig. 17 ON current sensitivity $\left(\Delta \boldsymbol{I}_{\boldsymbol{O}}\right)$ for four different asymmetric architectures 
Table 4 Results of sensitivity analysis due to process variation

\begin{tabular}{llllllll}
\hline Device & & $\begin{array}{l}V_{t h}(k=11) \\
(\mathrm{mV})\end{array}$ & $\begin{array}{l}I_{O N}(k=11) \\
(\mu \mathrm{A})\end{array}$ & $\begin{array}{l}V_{t h}(k=1) \\
(\mathrm{mV})\end{array}$ & $\begin{array}{l}I_{O N}(k=1) \\
(\mu \mathrm{A})\end{array}$ & $\begin{array}{l}\Delta V_{\text {th }} \\
(\mathrm{mV})\end{array}$ & $\begin{array}{l}\Delta I_{O N} \\
(\mu \mathrm{A})\end{array}$ \\
\hline Aligned & Source wide & 306.12 & 74.07 & 282.21 & 30.58 & 23.91 & 43.49 \\
Aligned & Drain wide & 319.97 & 72.86 & 273.51 & 33.34 & 46.46 & 39.52 \\
Missaligned & Source wide & 311.7 & 73.94 & 280.8 & 31.27 & 30.9 & 42.67 \\
Missaligned & Drain wide & 318.42 & 73.34 & 276.7 & 32.6 & 41.72 & 40.74 \\
\hline
\end{tabular}

profile in a confined region due to its liquid property like viscosity and surface tension. Influence of changing the degree of asymmetry on the biosensor performance and finding the solutions that can offer concave profiles has also been studied. The devices with wider drain side nanogap offers $\sim 100 \%$ and $\sim 40 \%$ higher $\Delta V_{t h}$ for aligned structure and misaligned structures respectively than its source side counterpart. Therefore, more the vertical $\mathrm{SiO}_{2}$ layers shift towards source end, stronger gate control and in turn, a higher sensitivity for a biosensor. In case of presently found SARS COV-2 virus (Coronavirus) detection, the nanogap structure and the dielectric constant should be modified and optimized according to the size of the Coronavirus (60-140 $\mathrm{nm}$ ) for better sensitivity and efficient detection with the same structure.

\section{Appendix}

$$
\begin{aligned}
P_{6}= & \frac{P_{4} \exp (L / \alpha)-P_{5}}{\exp (L / \alpha)-\exp (-L / \alpha)} \\
& +\frac{\exp (L / \alpha)-1}{\exp (L / \alpha)-\exp (-L / \alpha)}\left\{\frac{\alpha^{2} q N a}{\epsilon_{s i}}+\phi_{f b}\right\} \\
P_{7}= & \frac{\exp (L / \alpha)-1}{\exp (L / \alpha)-\exp (-L / \alpha)} \\
P_{8}= & P_{4}+\frac{\alpha^{2} q N a}{\epsilon_{s i}}+\phi_{f b}-P_{6} \\
P_{9}= & 1-P_{7} ; P_{10}=\frac{\alpha^{2} q N a}{\epsilon_{s i}}+\phi_{f b} \\
P_{11}= & 4 P_{7} P_{9}-1 \\
P_{12}= & 2 P_{10}+4 \phi_{f, s i}-4 P_{6} P_{9}-4 P_{7} P_{8} \\
P_{13}= & 4 P_{6} P_{8}-4 \phi_{f, s i}^{2}-P_{10}^{2}-4 \phi_{f, s i} P_{10}
\end{aligned}
$$

Acknowledgements We would like to thank the Department of ETCE, Jadavpur University for their constant support for providing the access of the laboratory.

Author Contributions Rahul Das: Conceptualization, Methodology, Software, Data curation, Writing, Reviewing. Ankush Chattopadhyay: Software, Writing-Original draft preparation, Methodology, Reviewing and Editing. Manash Chanda: Conceptualization, Reviewing and Editing. Chandan K. Sarkar: Supervision. Chayanika Bose:
Conceptualization, Methodology, Supervision, Correction of manuscript, Reviewing and Editing.

Funding No funding is available for this work.

Availability of data and materials Authors are not willing to disclose the data and materials related to this research, as this work is a part of thesis.

\section{Declarations}

Competing interests The authors declare that they have no known competing financial interests or personal relationships that could have appeared to influence the work reported in this paper.

Ethics Approval For this type of study formal consent is not required.

Consent to participate Not applicable for this type of study.

Consent to publication Not applicable for this type of study.

Research involving Human Participants and/or Animals Not applicable for this type of study.

Informed consent Not applicable for this type of study.

\section{References}

1. Oh SW, Moon JD, Lim HJ, Park SY, Kim T, Park JB, Han MH, Snyder M, Choi EY (2005) Calixarene derivative as a tool for highly sensitive detection and oriented immobilization of proteins in a microarray format through non-covalent molecular interaction. The FASEB Journal 19(10):1355-1337. https://doi.org/10. 1096/fj.04-2098fje

2. Drummond TG, Hill MG, Barton JK (2003) Electrochemical DNA sensors. Nature Biotechnology 21(10):1192-1199. https://doi.org/ 10.1038/nbt873

3. Fritz J, Baller MK, Lang HP, Rothuizen H, Vettiger P, Meyer E, Güntherodt H-J, Gerber C, Gimzewski JK (2000) Translating biomolecular recognition into nanomechanic. Science 288(5464):316-318. https://doi.org/10.1126/science.288.5464. 316

4. Huang X-J, Choi Y-K, Im H-S, Yarimaga O, Yoon E, Kim H-S (2006) Aspartate amino transferase (AST/GOT) and alanine aminotransferase (ALT/GPT) detection techniques. Sensors Basel Sensors 6(7):756-782

5. Yang YT, Callegari C, Feng XL, Ekinci KL, Roukes ML (2006) Zeptogram scale nano-mechanical mass sensing. Nanotechnology Letters 6(4):583-586. https://doi.org/10.1021/n1052134m 
6. Chen S, Bomer JG, Carlen ET, van den Berg A (2011) Al2O3/ silicon NanoISFET with near ideal nernstian response. Nano Letters 11:2334-2241. https://doi.org/10.1021/nl200623n

7. Knopfmacher O, Tarasov A, Fu W, Wipf M, Niesen B, Calame M, Schönenberger C (2010) Nernst limit in dual-gated si-nanowire FET sensors. Nano Letters 10(6):2268-2274. https://doi.org/10. 1021/nl100892y

8. Spijkman MJ, Brondijk JJ, Geuns TCT, Smits ECP, Cramer T, Zerbetto F, Stoliar P, Biscarini F, Blom PWM, de Leeuw DM (2010) Dual-gate organic field-effect transistors as potentiometric sensors in aqueous solution. Advance Functional Materials 20:898-905. https://doi.org/10.1002/adfm.200901830

9. Khamaisi B, Vaknin O, Shaya O, Ashkenasy N (2010) Electrical performance of silicon-on-insulator field-effect transistors with multiple top-gate organic layers in electrolyte solution. ACS Nano 4(8):4601-4608. https://doi.org/10.1021/nn100936h

10. Duarte-Guevara C, Lai F-L, Cheng C-W, Reddy B Jr, Salm E, Swaminathan V, Tsui Y-K, Tuan HC, Kalnitsky A, Liu Y-S, Bashir R (2014) Enhanced biosensing resolution with foundry fabricated individually addressable dual-gated ISFETs. Analytical Chemistry 86(16):8359-8367. https://doi.org/10.1021/ac501912x

11. Go J, Nair PR, Alam MA (2012) Theory of signal and noise in double-gated nanoscale electronic $\mathrm{pH}$ sensors. Journal of Applied Physics 112:034516. https://doi.org/10.1063/1.4737604

12. $1 \mathrm{~m} \mathrm{H}$, Huang X-1, Gu B, Choi Y-K, (2007) A dielectric- modulatedfield-effect transistor for biosensing. Nature Nanotechnology 2(7):430-434

13. Krahne R et al (2003) Nanoparticles and nanogaps: controlled positioning and fabrication. Physica E 17:498-502

14. Iqbal SM, Balasundaram G, Ghosh S, Bergstrom DE, Bashir R (2005) Direct currentelectrical characterization of ds-DNA in nanogap junctions. Applied Physics Letters 86:153901-153903

15. Ionescu-Zanetti C, Nevill JT, Carlo DD, Jeong KH, Lee LP (2006) Nanogap capacitors: sensitivity to sample permittivity changes. Journal of Applied Physics 99:024305-024309

16. Lazzarino $\mathrm{M}$ et al (2006) Twin cantilevers with a nanogap for single molecule experimentation. Microelectronic Engineering 83:1309-1311

17. Yi MQ, Jeong KH, Lee LP (2005) Theoretical and experimental study towards a nanogap dielectric biosensor. Biosensors and Bioelectronics 20:1320-1326

18. Narang R, Gupta M, Saxena M, Gupta RS (2012) Analytical model for a dielectric modulated double gate FET (DM-DG-FET) biosensor. In: 2012 International conference on emerging electronics, Mumbai, pp 1-4

19. Narang R, Reddy KVS, Saxena M, Gupta RS, Gupta M (2012) A dielectric-modulated tunnel-FET-based biosensor for label-free detection: analytical modeling study and sensitivity analysis. IEEE Transactions on Electron Devices 59(10):2809-2817. https://doi. org/10.1109/TED.2012.2208115
20. Narang R, Saxena M, Gupta M (2015) Comparative analysis of dielectric-modulated FET and TFET-based biosensor. IEEE Transactions on Nanotechnology 14(3):427-435. https://doi.org/ 10.1109/TNANO.2015.2396899

21. Choi J, Han J, Choi S, Choi Y (2010) Analytical modeling of a nanogap-embedded FET for application as a biosensor. IEEE Transactions on Electron Devices 57(12):3477-3484. https://doi. org/10.1109/TED.2010.2076152

22. Bhattacharyya A, Chanda M, De D (2019) Performance assessment of new dual-pocket vertical heterostructure tunnel FETbased biosensor considering steric hindrance issue. IEEE Transactions on Electron Devices 66(9):3988-3993. https://doi.org/10. 1109/TED.2019.2928850

23. Syu Y-C, Hsu W-E, Lin C-T (2018) Review-field-effect transistor biosensing: devices and clinical applications. ECS Journal of Solid State Science and Technology 7(7):3196-3207

24. Young KK (1989) Short-channel effect in fully depleted SOI MOSFETs. IEEE Transactions on Electron Devices 36(2):399_ 402. https://doi.org/10.1109/16.19942

25. Kang H, Han J, Choi Y (2008) Analytical threshold voltage model for double-gate MOSFETs with localized charges. IEEE Electron Device Letters 29(8):927-930. https://doi.org/10.1109/LED.2008. 2000965

26. Chattopadhyay A, Kundu A, Sarkar CK, Bose C (2020) Twodimensional modeling of the underlap graded-channel FinFET. Journal of Computational Electronics 19(2):688-699. https://doi. org/10.1007/s10825-020-01458-w

27. Liang X, Taur Y (2004) 2-D analytical solution for SCEs in DG MOSFETs. IEEE Trans Electron Devices 51(9):1385-1391. https://doi.org/10.1109/TED.2004.832707

28. Basak A, Sarkar A (2020) Drain current modelling of asymmetric junctionless dual material double gate MOSFET with high K gate stack for analog and RF performance. Silicon. https://doi.org/10. 1007/s12633-020-00783-w

29. Roldan JB, Gamiz F, Lopez-Villanueva JA, Carceller JE (1997) Modeling effects of electron velocity overshoot in a MOSFET. IEEE Transactions on Electron Devices 44(5):841-846

30. Chen YG, Kuo JB, Yu Z, Dutton RW (1995) An analytical drain current model for short-channel fully-depleted ultrathin silicononin-sulatornMOS devices. Solid State Electron 38(12):2051-2057

31. Sakurai T, Newton AR (1990) Alpha-power law MOSFET model and its applications to CMOS inverter delay and other formulas. IEEE Journal of Solid-State Circuits 25(2):584-594

32. Bhattacharyya AB (2009) Compact MOSFET models for VLSI design. Wiley, New York

Publisher's Note Springer Nature remains neutral with regard to jurisdictional claims in published maps and institutional affiliations. Silicon 